\title{
Chronic oral administration of calcium and iron to accelerate body lead detoxification after chronic lead exposure in Wistar rats Missoun Fatiha*
}

Address: University, Departement of Agronomy University of Mostaganem, Algeria

* Corresponding author

from International Society on Brain and Behaviour: 2nd International Congress on Brain and Behaviour Thessaloniki, Greece. 17-20 November 2005

Published: 28 February 2006

Annals of General Psychiatry 2006, 5(SuppI I):SI39 doi:I0.II86/I744-859X-5-SI-SI39

\begin{abstract}
Background
The toxic effects of lead are well-known. The nervous system and the haem synthesis are the two main points where the symptoms present themselves first. The common feature is that lead replaces $\mathrm{Zn}, \mathrm{Cu}, \mathrm{Fe}$, Ca cations or inhibits the enzymes related to these cations. The correlation between lead and essential metal $(\mathrm{Zn}, \mathrm{Cu}, \mathrm{Fe}, \mathrm{Ca})$ concentration in blood has been studied for several years. Not only these cations but some related proteins can regulate blood lead levels. We investigated essential metals in connection with lead detoxification, we reported that ingestion of calcium and iron in drinking water, may decrease intestinal absorption of $\mathrm{Pb}$ in rat wistar and we attempted to relate the behavioral effects of lead to neurotransmission.
\end{abstract}

\section{Materials and methods}

we evaluated the potential detoxifying effect of chronic oral $\mathrm{Pb}$ exposure in rats. For this purpose, four groups of seven young male Wistar rats weighing $45 \mathrm{~g}$ were treated as follows: group A received a semi-purified control diet for 5 weeks; groups B, C and D received the same diet plus $750 \mathrm{ppm}$ of pb (as acetate) for 5 weeks. Group B was then killed. We examined the blood samples taken. Groups C continued to receive the semi-purified control diet containing calcium $100 \mathrm{mg} / \mathrm{l}$ (as bicarbonate). Groups D receive the semi-purified control diet containing fer 10 $\mathrm{mg} / \mathrm{l}$ (as sulfate) for 5 additional weeks, Oral administration of $750 \mathrm{ppm}$ of lead acetate to young rats for 5 weeks caused a reduction in locomotor activity and stereotypic exploratory behavior during a 20 min testing period.

\section{Results}

This locomotor hypoactivity induced by lead was accompanied by a reduction in stereotypic behavior (sniffing, lickings, biting and grooming). Compared to the groups
A, B and C These outcomes suggested that lead might interfere with catecholaminergic and particularly dopaminergic neurotransmission. Rats were then killed we examined the blood samples taken

\section{Discussion}

The results showed that lead disturb haem synthesis of exposed rats compared to others groups. However the addition of calcium and iron administration was effective in decreasing $\mathrm{Pb}$ levels in rats. And help $\mathrm{Pb}$ detoxification. 